\title{
BMJ Open Aspirin and heparin for the prevention of pre-eclampsia: protocol for a systematic review and network meta- analysis
}

\author{
Jinzhu Huang, ${ }^{1}$ Xiaohong Chen, ${ }^{2}$ Haiyan Xing, ${ }^{1}$ Lin Chen, ${ }^{2}$ Zhaolu Xie, ${ }^{1}$ \\ Shuangshuang He, ${ }^{1}$ Xiaofang Wang, ${ }^{3}$ Yong Li, ${ }^{4}$ Huanhuan Cui, ${ }^{5}$ Jianhong Chen ${ }^{1}$
}

To cite: Huang J, Chen $X$, Xing $\mathrm{H}$, et al. Aspirin and heparin for the prevention of pre-eclampsia: protocol for a systematic review and network meta-analysis. BMJ Open 2019;9:e026920. doi:10.1136/ bmjopen-2018-026920

- Prepublication history and additional material for this paper are available online. To view these files, please visit the journal online (http://dx.doi. org/10.1136/bmjopen-2018026920).

$\mathrm{JH}, \mathrm{XC}$ and $\mathrm{HX}$ contributed equally.

Received 26 September 2018 Revised 14 January 2019 Accepted 16 January 2019

Check for updates

(C) Author(s) (or their employer(s)) 2019. Re-use permitted under CC BY-NC. No commercial re-use. See rights and permissions. Published by BMJ.

For numbered affiliations see end of article.

Correspondence to Professor Jianhong Chen; chenjhlab@126.com

\section{ABSTRACT}

Introduction Pre-eclampsia is an important cause of death and complication for pregnant women and perinatal infant. Low-dose aspirin has been most commonly used to prevent pre-eclampsia in high-risk pregnant women. Recently, heparins have also been used alone or in combination with aspirin to prevent pre-eclampsia. However, the optimal doses and combination therapy of aspirin and heparins are not well established. Therefore, we aim to compare aspirin, heparins and their combination to prevent pre-eclampsia in a network meta-analysis. Methods and analysis We will search the following electronic databases from the date of database establishment to 8 January 2019: PubMed, Embase, Cochrane Library, Web of Science and ProQuest. We will also search additional studies manually. There will be no restriction on the language of publications. Only randomised clinical trials will be eligible in our network meta-analysis. We will include pregnant women who have been recommended for aspirin according to the standard of the American Congress of Obstetricians and Gynecologists, or were designated as high risk in some recent studies. We will include studies comparing the effects of any single or combination of aspirin and heparins with placebo or observation or another intervention in pregnancy. We will include studies that reported one of the following outcomes: pre-eclampsia, severe pre-eclampsia, preterm delivery, perinatal death and full-term pre-eclampsia with delivery at $\geq 37$ weeks. Traditional pairwise meta-analysis will be performed initially, and then network meta-analysis will be performed using frequency analysis method. Subgroup analyses and sensitivity analyses will be conducted to assess the robustness of the findings.

Ethics and dissemination This network meta-analysis does not require ethical certification. An overview and information on the prevention of pre-eclampsia in high-risk pregnant women will be provided by this network metaanalysis.

PROSPERO registration number CRD42018084248.

\section{BACKGROUND}

Pre-eclampsia remains one of the most frequent complications among pregnant women in the clinic. It is a complex and
Strengths and limitations of this study

- This will be the first network meta-analysis to explore the optimal dose of aspirin and its combination therapy with heparins to prevent pre-eclampsia.

- We will include only randomised clinical trials.

- Selection, data extraction and bias assessment will be performed by two independent reviewers.

- Traditional pairwise meta-analysis and network meta-analysis will be performed simultaneously.

- A limitation of this network meta-analysis is whether the inclusion criteria used are sufficient.

multisystem disorder associated with significant morbidity and mortality in mothers and their fetus. ${ }^{1}$ Some aetiologies can increase the risk of this complication during pregnancy. Three risk categories have been classified by the American Congress of Obstetricians and Gynecologists (ACOG). ${ }^{2}$ History of pre-eclampsia, multifetal gestation, chronic hypertension, type 1 or 2 diabetes, renal disease and autoimmune disease (systemic lupus erythematosus or antiphospholipid syndrome) are high-risk factors for gravida. However, nulliparity, obesity (body mass index [BMI] greater than 30), family history of pre-eclampsia, sociodemographic characteristics (African-American race or low socioeconomic), age 35 years or older and personal history factors (low birth weight or small for gestational age, or previous adverse pregnancy outcome or more than 10-year pregnancy interval) always lead to moderate risks of pre-eclampsia. Previous uncomplicated full-term delivery is regarded as low risk. In addition to ACOG, other international organisations have also recommended their own standards. However, how risk assessment should be performed is still in dispute, and no international consensus has been reached. 
However, some measures have been taken to prevent pre-eclampsia, such as low-dose aspirin, unfractionated heparin (UFH), low molecular weight heparin (LMWH), calcium supplementation, antioxidants, low-salt diet and diuretic use. ${ }^{3}$ Currently, low-dose aspirin is the most widely prescribed treatment, and the dosages of aspirin used to prevent pre-eclampsia range from $50 \mathrm{mg} /$ day to $150 \mathrm{mg} /$ day. ${ }^{1}{ }^{4}$ Aspirin (or acetylsalicylic acid) is a cyclo-oxygenase inhibitor which has been used to treat or prevent diseases in many areas. Long-term daily use in patients might lead to an increased risk of adverse reactions, such as gastrointestinal and cerebral bleeding. Most adverse reactions are time-dependent and dose-dependent. Therefore, our assumption is that (1) lower dosage aspirin might be safer and more economical if it has the same efficacy as a higher dose and (2) an optimal dose of aspirin to prevent pre-eclampsia might be in the reported range. Unfortunately, the optimal dosage and timing of aspirin reported in numerous clinical trials and meta-analyses are diverse. ${ }^{5-10}$ Furthermore, there are few head-tohead comparative trials to explore the effectiveness between different doses of aspirin. Some conventional meta-analyses have reported a greater reduction in the risk of pre-eclampsia with the use of aspirin $100-150 \mathrm{mg} /$ day compared with a lower dosage..$^{5}$ However, these conclusions are not appropriate due to flaws in methodology, which does not apply indirect adjustment model. The diversity of included patients in different studies also leads to multifarious results. In addition, dipyridamole and heparins have been added to aspirin or compared with it in some studies with inconsistent results. Heparins prescribed without aspirin are also controversial. ${ }^{11-18}$ In addition, some serious adverse reactions could be caused by heparins, including heparin-induced immune reactions, haemorrhage, osteoporotic fractures or maternal death. ${ }^{19} \mathrm{Few}$ network meta-analyses were performed to discuss these problems.

Therefore, we plan to evaluate what dosage of aspirin is optimal, whether heparins alone are effective, whether heparins in addition to aspirin are more effective than monotherapy, and the optimal combination of aspirin and heparins. We will perform a systematic review and network meta-analysis of randomised controlled trials to search for the answers.

\section{METHODS}

This network meta-analysis has been prospectively registered in the PROSPERO database (CRD42018084248 Available from: http://www.crd.york.ac.uk/PROSPERO

/display_record.php?ID=CRD42018084248). The protocol was prepared using the Preferred Reporting Items for Systematic Review and Meta-analysis Protocols (see online supplementary file 1). The study started on
14 November 2017 and is expected to be completed by 31 December 2019.

\section{Criteria for included studies \\ Types of studies}

Only randomised clinical trials (RCTs) will be eligible in our network meta-analysis, and studies with other designs will not be eligible. Full-length articles or letters in peer-reviewed journals will be eligible. Registered clinical trials and conference papers will also be eligible. No language restrictions will be applied. Studies will not be considered due to unobtainable data.

\section{Types of participants}

How to identify pregnant women at high risk of pre-eclampsia is still a big problem. Therefore, who should be given drug prophylaxis is still being debated. Atallah and his coauthors ${ }^{4}$ have listed those who are high risk defined by six special organisations or national guidelines. These risk factors are (1) history of pre-eclampsia, (2) autoimmune disease (systemic lupus erythematosus, antiphospholipid syndrome), (3) diabetes (type 1 or 2), (4) renal disease, (5) chronic hypertension, (6) multifetal gestation and (7) association of moderate-risk factors. According to the standard of ACOG, women with $\geq 1$ high-risk factors or $\geq 2$ moderate-risk factors were classified as meeting the criteria for aspirin prophylaxis. ${ }^{20}$ Recently, a systematic review and meta-analysis of large cohort studies suggested that the presence of assisted reproductive technology and BMI $>30$ might suffice to designate a pregnant woman as 'high risk'. ${ }^{21}$ Similarly, some recent large trials are exclusive based on the use of multivariate screening algorithms rather than maternal history alone..$^{22-24}$

In this network meta-analysis, eligible studies should have met one of the following criteria: (1) the included participants had any one of high-risk or $\geq 2$ moderate-risk factors defined by ACOG, (2) the presence of assisted reproductive technology or BMI $>30$, and (3) the included participants were screened to predict pre-eclampsia using a combination of maternal factors and biomarkers. We will not include patients with abnormal uterine artery blood flow tested by uterine artery Doppler or other biomarkers alone (mean arterial pressure, serum placental growth factor and pregnancy-associated plasma protein A). Studies with only one medium-risk factor will also not be included. There are no restrictions on age and race of pregnant women. We will include studies on patients with any starting time of treatment.

\section{Types of interventions}

We will include studies comparing the effects of aspirin or heparins with placebo, observation or another intervention, and their combinations are also considered. Studies that compared different dosages of aspirin will be included, as well as RCTs comparing heparins with monotherapy in addition to aspirin. Heparins will include UFH and LMWH (enoxaparin, dalteparin, nadroparin). We will include studies with two or more treatment arms. 


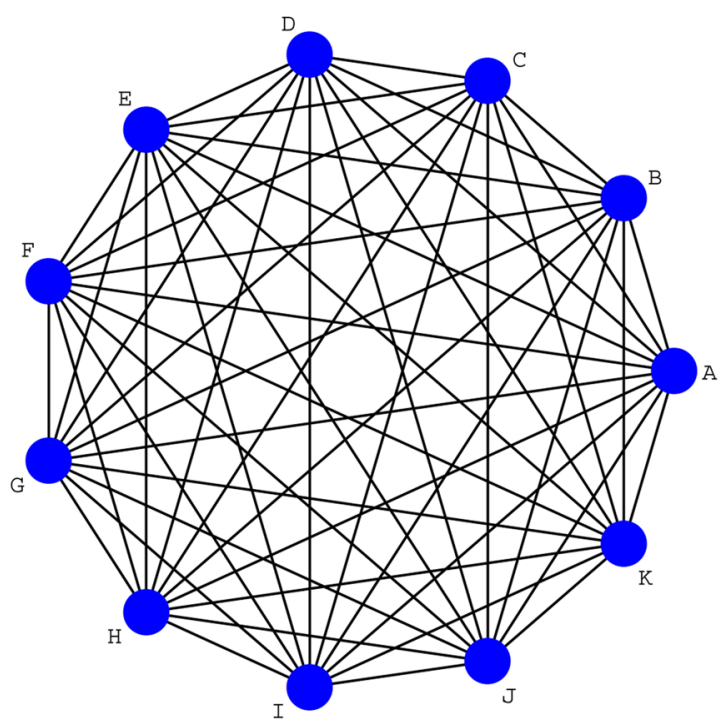

Figure 1 The ideal network plot based on expected eligible interventions. A: $50-81 \mathrm{mg} /$ day aspirin; B: $100 \mathrm{mg} /$ day aspirin; C: $150 \mathrm{mg} /$ day aspirin; D: unfractionated heparin (UFH); E: low molecular weight heparin (LMWH); F: 50-81 mg/day aspirin combined with UFH; G: $100 \mathrm{mg} /$ day aspirin combined with UFH; H: $150 \mathrm{mg} /$ day aspirin combined with UFH; I: 50-81 mg/day aspirin combined with LMWH; J: $100 \mathrm{mg} /$ day aspirin combined with LMWH; K: $150 \mathrm{mg} /$ day aspirin combined with LMWH.

The interventions or the exposures will be divided into 11 groups: 50-81 mg/day aspirin, $100 \mathrm{mg} /$ day aspirin, $150 \mathrm{mg}$ /day aspirin, UFH, LMWH, 50-81 mg/day aspirin combined with UFH, $100 \mathrm{mg} /$ day aspirin combined with UFH, $150 \mathrm{mg} /$ day aspirin combined with UFH, 50-81 mg/day aspirin combined with LMWH, $100 \mathrm{mg} /$ day aspirin combined with LMWH, and $150 \mathrm{mg} /$ day aspirin combined with LMWH. Comparator(s) or control group will be placebo, observation, another intervention, heparins or different dosages of aspirins. Of course, the studies classified in the same group must have homogeneity. An ideal network plot, which is a fully connected network with all the expected interventions, has been generated (figure 1).

Types of outcome measures

We will include studies that reported at least one of the following outcomes.

\section{Primary outcomes}

Pre-eclampsia (hypertension [blood pressure $\geq 140$ systolic and/or $\geq 90$ diastolic] with new-onset proteinuria [300 mg or more in 24 hours or two readings of at least++ on dipstick analysis] at or beyond 20 weeks' gestation).

\section{Secondary outcomes}

- Severepre-eclampsia (blood pressure $\geq 160$ systolicand/ or $\geq 110$ diastolic and/or proteinuria $\geq 5 \mathrm{~g} / 24$ hours).

- Preterm delivery (preterm birth at less than 37 weeks' gestation and infant small for gestational age at birth).
- Perinatal death (death in uterus or death of the baby before discharge from the hospital).

- Full-term pre-eclampsia with delivery at $\geq 37$ weeks.

\section{Patient and public involvement}

This systematic review and network meta-analysis is based on published randomised controlled trials, so primary patients' data will not be collected. Patients and the public are not involved in the study design, recruitment and data analysis.

\section{Data sources and search strategy}

Literature search will be performed mainly in five databases: PubMed, Embase, Cochrane Library, Web of Science and ProQuest. We will search additional studies manually (International Clinical Trials Registry Platform [http://www.who.int/ictrp/en/], clinical trials [https://www.clinicaltrials.gov/], American Journal of Obstetrics and Gynecology [AJOG; https://www.ajog.org/], Obstetrics E Gynecology [OG; https://journals.lww.com/ greenjournal/pages/default.aspx], and BJOG: An International Journal of Obstetrics $\mathcal{E}$ Gynaecology [http://www. bjog.org/view/0/index.html]). The studies that have been included in the published systematic reviews and meta-analyses are also supplemented into this study, if they are not searched by other methods. The database was searched from the date of establishment to 8 January 2019, and three journals from 2016 to 2019 ( $A J O G, O G$ and $B J O G$ ) will be searched. There will be no restriction on the language of publications. We will attempt to contact the authors by emails if we cannot find the unavailable studies from library resources sharing (http://www.yz365.com/). If data of the studies are not available, we will exclude the study. The search strategy has been described in online supplementary file 2.

\section{Data collection and analysis}

\section{Selection of studies}

Titles and abstracts of the searched studies will be imported into EndNote V.X5.0.1, then JH and XC will independently screen and select. The trials will be excluded if two investigators judge that the studies do not meet the inclusion criteria. We will also obtain full texts if the trials cannot be identified by titles and abstracts. The inter-rater reliability of both reviewers will be calculated by a pilot test (see online supplementary file 3). Any discrepancies will be discussed among four reviewers $(\mathrm{JH}$, $\mathrm{XC}, \mathrm{HX}, \mathrm{JC}$ ), and the trials will be included if three-quarters of the reviewers reach consensus.

\section{Data extraction and management}

The data of included studies will be extracted into the predetermined sheet and then entered into Microsoft Excel V.2010. Two investigators will independently (JH, $\mathrm{XC}$ ) extract the following information according to the predetermined table: study design, patients' characteristics (age, gestational age at entry, risk factors), interventions, comparisons, outcomes, inclusion criteria, 
exclusion criteria and diagnostic criteria. The table is found in online supplementary file 4 .

\section{Risk of bias assessment}

The methodological quality of eligible trials will be evaluated independently by two investigators $(\mathrm{JH}, \mathrm{XC})$ using 'the Cochrane Collaboration's tool for assessing risk of bias' (random sequence generation, allocation concealment, blinding of participants and personnel, blinding of outcome assessment, incomplete outcome data, selective reporting, and other sources of bias). ${ }^{25}$ Disagreements between the two authors will be resolved by discussion. If the disagreement persists, a senior investigator (LC or JC) will be consulted to reach consensus. The quality of each eligible study will be assessed using RevMan V.5.1.0.

\section{Statistical analysis}

Traditional pairwise meta-analysis will be performed using Stata V.14.0 software. The pooled estimates of relative risks (RRs) and 95\% CIs of direct comparisons between two strategies will be calculated using random-effects or fixed-effects model. The RRs will be directly pooled across studies using fixed-effect model if heterogeneity is absent, otherwise a random-effect model will be used. Network meta-analysis will be performed using frequency analysis method and Stata V.14.0 software. Network evidence and contribution diagram will be established. Finally, a predictive probability of the best intervention will be estimated using the surface under a cumulative ranking curve. ${ }^{26}$ Efficacy of preventive measures will be ranked by predicting probability.

\section{Dealing with missing data}

The intention-to-treat principle will be used to deal with missing count data. The dropouts will be considered to be non-responders if they drop after the randomisation. To obtain missing data, we will first attempt to contact the authors by emails. Otherwise, the data will be verified from other trials in the network or from other published meta-analyses. ${ }^{27}$

\section{Measures for transitivity assumption}

Clinical and methodological similarities are most commonly used to assess transitivity between eligible trials. Similarities in clinical factors mainly include baseline characteristics of patients (ie, population, intervention and comparison characteristics), follow-up time and clinical outcomes. The design and quality of each eligible study are methodological similarities. ${ }^{28}$ We will assess transitivity of the included studies according to the above factors.

\section{Investigation of heterogeneity}

$\mathrm{I}^{2}$ statistics will be used to assess heterogeneity among studies. We consider the value of $\mathrm{I}^{2}$ from $0 \%$ to $24 \%$, $25 \%-50 \%$ and greater than $50 \%$ as low, moderate and high heterogeneity. ${ }^{29}$ Meta-regression model will be used to explore reasons, if heterogeneity is moderate and high.

\section{Measures for inconsistency}

A design-treatment interaction model will be used to check and explore inconsistency assumption. ${ }^{30}$ An inconsistency factor (IF) will then be estimated. IF will be assessed by $\mathrm{p}$ value and Z-test. $\mathrm{P}$ values $<0.05$ will be considered statistically significant.

\section{Measures for publication bias}

Publication bias will be examined with the funnel plot method, the Begg's adjusted rank correlation test and the Egger's regression asymmetry test. In addition, small study effect for the whole network will be assessed by constructing a comparison-adjusted funnel plot taking into account different comparisons. ${ }^{31}$ In the absence of small study effects, the studies will form an inverted funnel centred at 0 .

\section{Subgroup analyses and sensitivity analyses}

We will perform subgroup analyses according to the results of heterogeneity and inconsistency, if sufficient data are available. Influence analysis is used to assess how a single study affects the combined effect amount. We will perform sensitivity analyses by this method.

\section{Ethics and dissemination}

The findings of this analysis will provide an overview and information on the prevention of pre-eclampsia in highrisk pregnant women. It is hoped that the findings will have significant implications for clinical practice and provide bases for further research.

\section{Author affiliations}

${ }^{1}$ Department of Pharmacy, Daping Hospital, Third Military Medical University (Army Medical University), Chongqing, China

${ }^{2}$ Department of Pharmacy, Chongqing Health Center for Women and Children, Chongqing, China

${ }^{3}$ Department of Laboratory Medicine, Key Laboratory of Diagnostic Medicine (Ministry of Education), Chongqing Medical University, Chongqing, China ${ }^{4}$ Department of Pharmacy, Puai Hospital, Tongji Medical College, Huazhong University of Science and Technology, Wuhan, China

${ }^{5}$ Centre for Drug Evaluation, China Food and Drug Administration, Beijing, China

Acknowledgements We thank Emmanuel Bujold from Laval University, Canada, Jon Hyett from Royal Prince Alfred Hospital, University of Sydney, Australia, for their valuable advice. Jon Hyett was involved in the meta-analysis in reference 7 and he has no competing interests.

Contributors $\mathrm{JH}, \mathrm{XC}, \mathrm{HX}, \mathrm{LC}$ and $\mathrm{JC}$ conceived the study and drafted the protocol. $\mathrm{JH}, \mathrm{XC}, \mathrm{HX}$ and $\mathrm{JC}$ wrote the first draft of the manuscript. XW, SH, ZX, YL, HC and LC assisted in protocol design and revision. $\mathrm{JH}, \mathrm{XC}$ and $\mathrm{XW}$ participated in the search strategy development. ZX, YL and HC participated in the design of data synthesis and analysis. All the authors approved the publication of the protocol.

Funding This research received no specific grant from any funding agency in the public, commercial or not-for-profit sectors.

Competing interests Jon Hyett from Royal Prince Alfred Hospital, University of Sydney, Australia, was involved in the meta-analysis referenced No.7.

Patient consent for publication Not required.

Ethics approval This network meta-analysis does not require formal ethical approval as primary patients' data will not be collected. 
Provenance and peer review Not commissioned; externally peer reviewed.

Open access This is an open access article distributed in accordance with the Creative Commons Attribution Non Commercial (CC BY-NC 4.0) license, which permits others to distribute, remix, adapt, build upon this work non-commercially, and license their derivative works on different terms, provided the original work is properly cited, appropriate credit is given, any changes made indicated, and the use is non-commercial. See: http://creativecommons.org/licenses/by-nc/4.0/.

\section{REFERENCES}

1. Katsi V, Kanellopoulou T, Makris T, et al. Aspirin vs heparin for the prevention of preeclampsia. Curr Hypertens Rep 2016;18:1-8.

2. Low-dose aspirin use during pregnancy. ACOG committee opinion no. 743: low-dose aspirin use during pregnancy. Obstet Gynecol 2018;132:e44-52.

3. Jim B, Karumanchi SA. Preeclampsia: pathogenesis, prevention, and long-term complications. Semin Nephrol 2017;37:386-97.

4. Atallah A, Lecarpentier E, Goffinet F, et al. Aspirin for prevention of preeclampsia. Drugs 2017;77:1819-31.

5. Roberge S, Bujold E, Nicolaides KH. Aspirin for the prevention of preterm and term preeclampsia: systematic review and metaanalysis. Am J Obstet Gynecol 2018;218:287-93.

6. Meher S, Duley L, Hunter K, et al. Antiplatelet therapy before or after 16 weeks' gestation for preventing preeclampsia: an individual participant data meta-analysis. Am J Obstet Gynecol 2017;216:121-8.

7. Roberge S, Nicolaides K, Demers S, et al. The role of aspirin dose on the prevention of preeclampsia and fetal growth restriction: systematic review and meta-analysis. Am J Obstet Gynecol 2017;216:110-20.

8. Xu TT, Zhou F, Deng CY, et al. Low-dose aspirin for preventing preeclampsia and its complications: a meta-analysis. J Clin Hypertens 2015;17:567-73.

9. Villa PM, Kajantie E, Räikkönen K, et al. Aspirin in the prevention of pre-eclampsia in high-risk women: a randomised placebo-controlled PREDO Trial and a meta-analysis of randomised trials. BJOG 2013;120:64-74.

10. Roberge $S$, Giguère $Y$, Villa $P$, et al. Early administration of lowdose aspirin for the prevention of severe and mild preeclampsia: a systematic review and meta-analysis. Am J Perinatol 2012;29:551-6.

11. Beaufils M, Uzan S, Donsimoni R, et al. Prevention of pre-eclampsia by early antiplatelet therapy. Lancet 1985;1:840-2.

12. Goel N, Tuli A, Choudhry R. The role of aspirin versus aspirin and heparin in cases of recurrent abortions with raised anticardiolipin antibodies. Med Sci Monit 2006;12:CR132-6.

13. Rey E, Garneau P, David M, et al. Dalteparin for the prevention of recurrence of placental-mediated complications of pregnancy in women without thrombophilia: a pilot randomized controlled trial. $J$ Thromb Haemost 2009;7:58-64.

14. Gris JC, Chauleur C, Faillie JL, et al. Enoxaparin for the secondary prevention of placental vascular complications in women with abruptio placentae. The pilot randomised controlled NOH-AP trial. Thromb Haemost 2010;104:771-9.

15. Visser J, Ulander VM, Helmerhorst FM, et al. Thromboprophylaxis for recurrent miscarriage in women with or without thrombophilia.
HABENOX: a randomised multicentre trial. Thromb Haemost 2011;105:295-301.

16. de Vries JI, van Pampus MG, Hague WM, et al. Low-molecularweight heparin added to aspirin in the prevention of recurrent earlyonset pre-eclampsia in women with inheritable thrombophilia: the FRUIT-RCT. J Thromb Haemost 2012;10:64-72.

17. Martinelli I, Ruggenenti P, Cetin I, et al. Heparin in pregnant women with previous placenta-mediated pregnancy complications: a prospective, randomized, multicenter, controlled clinical trial. Blood 2012;119:3269-75.

18. Rodger MA, Hague WM, Kingdom J, et al. Antepartum dalteparin versus no antepartum dalteparin for the prevention of pregnancy complications in pregnant women with thrombophilia (TIPPS): a multinational open-label randomised trial. Lancet 2014;384:1673-83.

19. Deruelle P, Coulon C. The use of low-molecular-weight heparins in pregnancy--how safe are they? Curr Opin Obstet Gynecol 2007;19:573-7.

20. Al-Rubaie ZTA, Askie LM, Hudson HM, et al. Assessment of NICE and USPSTF guidelines for identifying women at high risk of preeclampsia for tailoring aspirin prophylaxis in pregnancy: an individual participant data meta-analysis. Eur J Obstet Gynecol Reprod Biol 2018;229:159-66.

21. Bartsch E, Medcalf KE, Park AL, et al. Clinical risk factors for preeclampsia determined in early pregnancy: systematic review and meta-analysis of large cohort studies. BMJ 2016;353:i1753.

22. O'Gorman N, Wright D, Syngelaki A, et al. Competing risks model in screening for preeclampsia by maternal factors and biomarkers at 11-13 weeks gestation. Am J Obstet Gynecol 2016;214:103.e1-12.

23. O'Gorman N, Wright D, Poon LC, et al. Accuracy of competingrisks model in screening for pre-eclampsia by maternal factors and biomarkers at 11-13 weeks' gestation. Ultrasound Obstet Gynecol 2017; $49: 751-5$

24. Rolnik DL, Wright D, Poon LC, et al. Aspirin versus placebo in pregnancies at high risk for preterm preeclampsia. N Engl J Med 2017;377:613-22.

25. Higgins JPT GS. Cochrane handbook for systematic reviews of interventions Version 5.1.0: The Cochrane Collaboration, 2011.

26. Salanti G, Ades AE, Ioannidis JP. Graphical methods and numerical summaries for presenting results from multiple-treatment metaanalysis: an overview and tutorial. J Clin Epidemiol 2011;64:163-71.

27. Furukawa TA, Barbui C, Cipriani A, et al. Imputing missing standard deviations in meta-analyses can provide accurate results. J Clin Epidemiol 2006;59:7-10.

28. Salanti G. Indirect and mixed-treatment comparison, network, or multiple-treatments meta-analysis: many names, many benefits, many concerns for the next generation evidence synthesis tool. Res Synth Methods 2012;3:80-97.

29. Huang J, Wang $X$, Chen $X$, et al. Perioperative antibiotics to prevent acute endophthalmitis after ophthalmic surgery: a systematic review and meta-analysis. PLoS One 2016;11:e0166141.

30. Higgins JP, Jackson D, Barrett JK, et al. Consistency and inconsistency in network meta-analysis: concepts and models for multi-arm studies. Res Synth Methods 2012;3:98-110.

31. Chaimani A, Salanti G. Using network meta-analysis to evaluate the existence of small-study effects in a network of interventions. Res Synth Methods 2012;3:161-76. 